\title{
Simulation Analysis of Balance Detection Technique in Coherent Optical Receiver
}

\section{Piaokun Zhang, Zhongwei Tan*, Zhichao Ding, Lijun Guo}

Key Laboratory of All Optical Network \& Advanced Telecommunication Network of EMC, Institute of Lightwave Technology, Beijing Jiaotong University, Beijing, China

Email: 19125071@bjtu.edu.cn, *zhwtan@bjtu.edu.cn,17111006@bjtu.edu.cn,19120052@bjtu.edu.cn

How to cite this paper: Zhang, P.K., Tan, Z.W., Ding, Z.C. and Guo, L.J. (2021) Simulation Analysis of Balance Detection Technique in Coherent Optical Receiver. Optics and Photonics Journal, 11, 301-313. https://doi.org/10.4236/opj.2021.118020

Received: March 23, 2021

Accepted: July 31, 2021

Published: August 3, 2021

\begin{abstract}
This paper introduces the working principle of the balanced heterodyne detection system, establishes the corresponding mathematical model, deduces the signal to noise ratio (SNR) formula of the balanced heterodyne detection. By comparing balance heterodyne detection with general coherent detection with MATLAB numerical simulation, the superiority of balance heterodyne detection system is proved theoretically. Finally, the simulation models of ordinary heterodyne detection, balance detection and double balance detection system are built by OptiSystem. The simulation results are consistent with the conclusions derived from the mathematical analysis, which provides a new method for the research of weak laser detection technology.
\end{abstract}

\section{Keywords}

Balance Heterodyne Detection, $90^{\circ}$ Optical Mixer, Signal to Noise Ratio, The Numerical Simulation

\section{Introduction}

As early as the 1980s, optical coherence detection technology has been focusing in the field of optical communication [1]. The balance detection and double balance detection system were proposed during this period [2]. In 1985, Gregory et al. proposed the concept of balanced detection, that two photoelectric detectors with nearly identical parameters were simultaneously used in a photoelectric detection unit, and the signal light and local oscillator light were incident into the two photoelectric detectors respectively. Then the high-frequency signals were filtered through the difference circuit to obtain the required medium-frequency signals [3]. The balance heterodyne detection overcomes the disadvantage of low utilization rate of local oscillator in single tube detection, 
which allows that the coherent optical communication receiver obtains high sensitivity and SNR at lower local oscillator power. The balance heterodyne detection can also effectively suppress the interference of common mode noise and improve the SNR of coherent optical communication system close to the quantum noise limit level [4]. The double balance detection technology was developed by Kuwaharra et al. on the basis of the balance detection theory in 1987. It not only has the advantages of the balance detection technology, but also has a variety of modulation and demodulation methods, as well as good environmental adaptability and better system stability [5].

In this paper, through the derivation of the mathematical model of balance detection, MATLAB is used to analyze the SNR of the balance detection and ordinary heterodyne detection. In addition, optical simulation software OptiSystem is used to build the simulation models of ordinary heterodyne detection, balance detection and double balance detection system.

\section{Theoretical Derivation}

\subsection{Mathematical Model of Balance Detection Technique}

As we all know, for IM/DD system directly incident light field on the photodetector only signal light $E_{S}(t)$, so the output of the detector should be:

$$
S_{P D}=i_{P D}^{2} R_{L}=\beta^{2} P_{S}^{2} R_{L} .
$$

In Equation (1), $\beta$ is the responsiveness of the photodetector.

Suppose the signal light of heterodyne detection is $E_{S}(t)=A_{s} \cos \left(\omega_{S}(t)+\phi_{s}\right)$, The local oscillator light is $E_{L}(t)=A_{L} \cos \left(\omega_{L}(t)+\phi_{L}\right)$, Then the output of ordinary heterodyne detection is [6]:

$$
\begin{aligned}
& i_{P D}=\beta \overline{\left[E_{S}(t)+E_{L}(t)\right]^{2}}=\beta\left\{A_{S}^{2} \overline{\cos ^{2}\left(w_{s} t+\phi_{s}\right)}+A_{L}^{2} \overline{\cos ^{2}\left(w_{L} t+\phi_{L}\right)}\right. \\
& \left.+A_{s} A_{L} \overline{\cos \left[\left(w_{s}+w_{L}\right) t+\left(\phi_{s}+\phi_{L}\right)\right]}+A_{s} A_{L} \overline{\cos \left[\left(w_{s}-w_{L}\right) t+\left(\phi_{s}-\phi_{L}\right)\right]}\right\} .
\end{aligned}
$$

Due to the limitation of intermediate frequency bandwidth, the photodetector can only respond to the difference frequency term, then the final detected current signal is [7]:

$$
i_{\text {out }}=\beta A_{s} A_{L} \cos \left[\left(w_{s}-w_{L}\right) t+\left(\phi_{s}-\phi_{L}\right)\right] .
$$

The principle of balance detection is shown in Figure 1. After the signal light $E_{s}$ and the local oscillator light $E_{L}$ are input to the beam splitter B through the optical fiber, two signals lights $\left(E_{s 1}\right.$ and $\left.E_{s 2}\right)$ and two local oscillator lights $\left(E_{L 1}\right.$ and $E_{L 2}$ ) are generated respectively. Where $E_{S 1}$ and $E_{L 1}$ are received by photodetector 1 and output photocurrent i1; $E_{s 2}$ and $E_{L 2}$ are received by photodetector 2 and the output current is i2. The phase of the photocurrent output by the two detectors is opposite, and the final IF signal is obtained after the difference frequency.

It is known that the signal light of heterodyne detection is $E_{S}(t)=E_{S} \cos \left(\omega_{S}(t)+\phi_{S}\right)$, and the local oscillator light is 


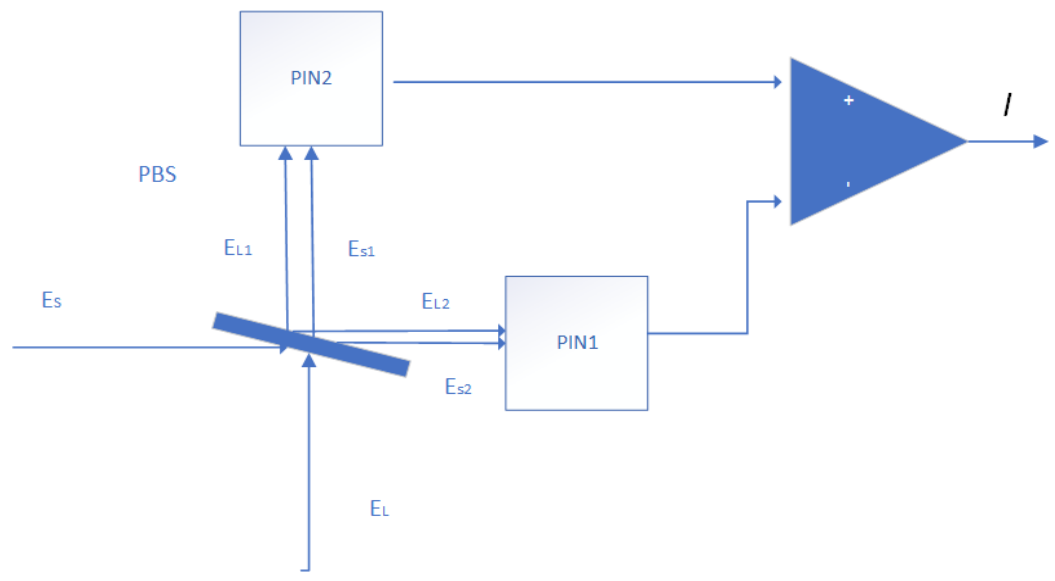

Figure 1. Schematic diagram of balance detection.

$$
E_{L}(t)=E_{L} \cos \left(\omega_{L}(t)+\phi_{L}\right)
$$

where: $E_{S}, \omega_{S}, \phi_{S}$ and $E_{L}, \omega_{L}, \phi_{L}$ are the amplitude, frequency and phase of the signal light and the local oscillator light respectively. Assuming that the fraction ratio of the beam splitter is $\varepsilon$, the quantum efficiency of the two detectors is $\eta_{1}$ and $\eta_{2}$, and the bandwidth $\Delta f$ is equal, the energy of the signal light and the local oscillator light after passing through the beam splitter can be calculated as [8] [9]:

$$
\begin{aligned}
& E_{s_{1}}=\sqrt{1-\varepsilon} E_{s} \cos \left(\omega_{s}(t)+\phi_{s}\right) \\
& E_{s_{2}}=\sqrt{\varepsilon} E_{s} \cos \left(\omega_{S}(t)+\phi_{S}\right) \\
& E_{L_{2}}=\sqrt{1-\varepsilon} E_{L} \cos \left(\omega_{L}(t)+\phi_{L}\right) \\
& E_{L_{1}}=\sqrt{\varepsilon} E_{L} \cos \left(\omega_{L}(t)+\phi_{L}\right)
\end{aligned}
$$

The output of the four-way signal after mixing with the photodetector is:

$$
\begin{aligned}
i_{1}= & \frac{\eta_{1} e}{h \omega}\left\{(1-\varepsilon) E_{s}^{2}+\varepsilon E_{L}^{2}-2 \sqrt{1-\varepsilon} \sqrt{\varepsilon}\right. \\
& \left.\times E_{s} E_{L} \cos \left[\left(\omega_{s}-\omega_{L}\right) t+\phi_{s}-\phi_{L}\right]\right\}+n_{1} \\
i_{2}= & \frac{\eta_{2} e}{h \omega}\left\{\varepsilon E_{s}^{2}+(1-\varepsilon) E_{L}^{2}+2 \sqrt{1-\varepsilon} \sqrt{\varepsilon}\right. \\
& \left.\times E_{s} E_{L} \cos \left[\left(\omega_{s}-\omega_{L}\right) t+\phi_{s}-\phi_{L}\right]\right\}+n_{2}
\end{aligned}
$$

In the Equation (4), $n_{1}(t)$ and $n_{2}(t)$ are noise currents. The response current signal of the final balanced detection system is as follows:

$$
\begin{aligned}
i=i_{2}-i_{1}= & \frac{e}{h \omega}\left\{\left[\varepsilon \eta_{2}-(1-\varepsilon) \eta_{1}\right] E_{s}^{2}+\left[(1-\varepsilon) \eta_{2}-\varepsilon \eta_{1}\right] E_{L}^{2}\right\} \\
& +\frac{e}{h \omega}\left\{2\left(\eta_{1}+\eta_{2}\right) \sqrt{1-\varepsilon} \sqrt{\varepsilon} E_{s} E_{L} \times \sin \left[\left(\omega_{s}-\omega_{L}\right) t+\phi_{s}-\phi_{L}\right]\right\} \\
& +\left\{n_{2}(t)-n_{1}(t)\right\} .
\end{aligned}
$$

The double balance detection system is based on the structure of the balance heterodyne detection system, which uses four almost identical photoelectric de- 
tectors to form two groups of balance heterodyne detectors at the receiving end [10] [11] [12]. The photocurrent signal received by the detector is processed by differential processing to obtain the difference frequency signal. Its principle block diagram is shown in Figure 2.

The following mathematical model is used to illustrate this process.

Let the mathematical expression of the local oscillator light and the signal light be as follows:

$$
\begin{aligned}
E_{L} & =\left[\begin{array}{l}
k_{1} \\
k_{2}
\end{array}\right] \exp (i \phi) \\
E_{S} & =\left[\begin{array}{l}
k_{3} \\
k_{4}
\end{array}\right] \exp \left(i \omega_{i f} t\right)
\end{aligned}
$$

where, $k_{1}, k_{2}, k_{3}$ and $k_{4}$ represent the respective components of the local oscillator light and the signal light in two directions respectively.

Let $E_{L}^{\prime}$ be the circularly polarized light formed after the local oscillator light passes through the $1 / 4$ wave plate, then:

$$
E_{L}^{\prime}=\left[\begin{array}{c}
k_{1} \exp (i \pi / 2) \\
k_{2}
\end{array}\right] \exp [i(\phi-\pi / 4)]
$$

After the two beams of light pass through the beam splitting prism, they are divided into two beams of light in the horizontal and vertical directions, and the beam splitting prism transmits and reflects the matrix.

$$
T=\left[\begin{array}{cc}
t_{\|} \exp \left(i \tau_{1}\right) & 0 \\
0 & 0
\end{array}\right], R=\left[\begin{array}{cc}
0 & 0 \\
0 & r_{\perp} \exp \left(i \rho_{\perp}\right)
\end{array}\right] .
$$

It can be obtained from the above equation.

$$
\begin{aligned}
& E_{1}=\left[\begin{array}{c}
t_{1} \exp \left(i \tau_{\|}\right) k_{1} \exp [i(\phi+\pi / 4)] \\
r_{\perp} \exp \left(i \rho_{\perp}\right) k_{4} \exp \left(i \omega_{i f} t\right)
\end{array}\right] \\
& E_{2}=\left[\begin{array}{c}
t_{1} \exp \left(i \tau_{\|}\right) k_{3} \exp \left(i \omega_{i f} t\right) \\
r_{\perp} \exp \left(i \rho_{\perp}\right) k_{2} \exp [i(\phi+\pi / 4)]
\end{array}\right]
\end{aligned}
$$

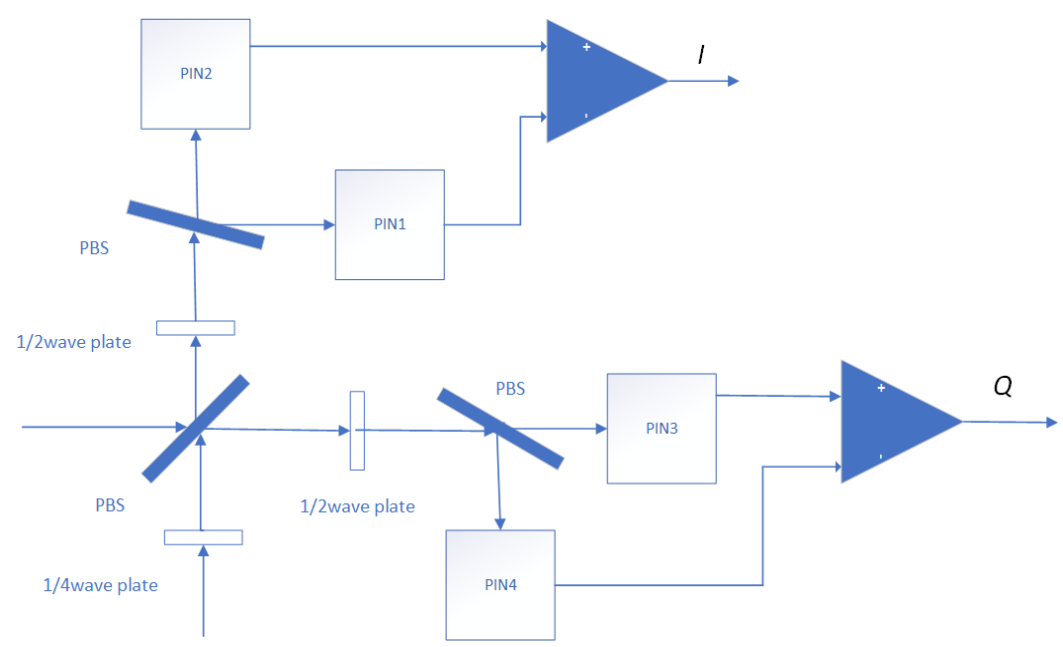

Figure 2. Schematic diagram of double balance detection. 
The transmission matrix of $1 / 2$ wave plate in the system is

$$
\Lambda_{1 / 2}=\frac{\sqrt{2}}{2}\left[\begin{array}{cc}
1 & 1 \\
1 & -1
\end{array}\right] \text {. }
$$

In Equation (10), the angle between the wave plate and the $\mathrm{X}$-axis is $22.5^{\circ}$.

Two beams of light through the beam splitter are divided into four different directions of the light signals, the photodetector after detection of the four channels phase difference $\pi / 2$ photocurrent signal.

$$
I_{0}=\frac{1}{2}\left\{k_{1}^{2}+k_{4}^{2}+2 k_{1} k_{4} \cos \left[\omega_{i f} t+\rho_{\perp}-\tau_{\|}-(\phi+\pi / 4)\right]\right\} .
$$

Similarly, the expressions of I90, I180 and I270 can be derived, which will not be expanded in detail here. Four channels of signal for differential processing, obtained two groups of phase difference $\pi / 2$ differential signals, namely orthogonal component $I$ and vertical component $Q$.

$$
\begin{aligned}
& I: I_{0}-I_{180}=2 k_{1} k_{4} \cos \left[\omega_{i f}-(\phi+\pi / 4)\right] \\
& Q: I_{90}-I_{270}=2 k_{2} k_{3} \sin \left[\omega_{i f}-(\phi+\pi / 4)\right]
\end{aligned}
$$

Compared with the direct detection method (Equation (1)), the ordinary heterodyne detection (Equation (3)) has the advantage of realizing the detection of the amplitude, frequency and phase of the signal light and improving the detection accuracy. It's good for detecting weak signals. According to the formula of photocurrent, it can be seen that the size of the photocurrent is proportional to the square root of the product of the power of the signal light and the local oscillator light. Therefore, when detecting weak signal, even if the signal light is very weak, as long as the local oscillator light power is large enough, a better signal can still be obtained eventually.

From Equation (5), it can be seen that balanced detection has advantages over single-source detection: on the one hand, it eliminates the DC component in the signal, which is convenient for signal processing, on the other hand, the amplitude of the AC component is double that of the output current of the single-source detector. In addition, due to the differential amplification used in the balanced detection, the common mode noise signals in the two detectors are greatly suppressed, and the signal-to-noise ratio of the output signals is improved. Dual balance detection not only has the advantage of balance detection, but also has a variety of modulation and demodulation methods, so its application prospect is very broad.

\subsection{Link Noise Analysis}

There are three types of noise in microwave photonic link: thermal noise, excess intensity noise and shot noise. The characteristics of each noise are analyzed in detail in the following sections [13].

Thermal noise is the noise caused by the Brownian motion of free electrons in the resistor. It has randomness and can only be increased but not eliminated. Therefore, in the balanced detection system, the thermal noise is the superposi- 
tion of the thermal noise of the two detectors. If the resistance of the two detectors is equal, the thermal noise power is:

$$
p_{\text {th }}=4 k T \Delta f \text {. }
$$

where $k$ is Boltzmann constant, $T$ is the temperature, $\Delta f$ is the bandwidth of the receiver.

Excess intensity noise is caused by fluctuations in local oscillator light output intensity and is usually described as relative intensity noise (RIN):

$$
R I N=10 \log \frac{\left\langle p_{\mathrm{N}}^{2}\right\rangle}{\langle p\rangle^{2} \Delta f} .
$$

where $\left\langle p_{\mathrm{N}}^{2}\right\rangle$ is the mean square value of noise power, $\langle p\rangle^{2}$ is the average output power of the laser, $\Delta f$ is the bandwidth of the receiver. The unit of relative intensity noise is $\mathrm{dBc} / \mathrm{Hz}$. It can be seen that the mean square value of laser noise power is proportional to the relative intensity noise, and the value range of relative intensity noise is $-160--120 \mathrm{dBc} / \mathrm{Hz}$ [14].

$$
\begin{gathered}
p_{\text {rin }}=2 e \gamma I^{2}{ }_{\mathrm{dc}} \Delta f . \\
2 e \gamma=10^{\frac{R I N}{10}} .
\end{gathered}
$$

where, $\gamma$ represents the variation of noise power of the local oscillator with the square of the average power of the local oscillator. The unit is $\mathrm{A}^{-1}$, and its corresponding value range is $10^{2}-10^{6} \mathrm{~A}^{-1}$.

Assuming that the bandwidth of the two detectors is the same, since the excess intensity noise incident to the two detectors respectively is related, the square value of the excess intensity noise current of the balanced detector is [15]:

$$
p_{\text {rin }}=2 e \gamma\left[\eta_{1} \varepsilon E_{1}^{2}-\eta_{2}(1-\varepsilon) E_{1}^{2}\right] \Delta f .
$$

Shot noise is caused by the random fluctuation of photoelectric current in the photodetector. The shot noise current is a random process of Poisson distribution. The power spectral density of the shot noise is flat, so for the balanced detection system, the shot noise of the two signals after differential processing can be expressed as:

$$
p_{\mathrm{sh}}=2 e I_{D C} \Delta f=2 e\left(I_{D C 1}+I_{D C 2}\right) \Delta f .
$$

Therefore, the SNR of the balanced detection method is:

$$
\begin{aligned}
& S N R_{\text {balance }}=\frac{i_{\text {signal }}^{2}}{p_{\text {sh }}+p_{\text {rin }}+p_{\text {th }}} \\
& =\frac{2 e^{2} E_{S}^{2} E_{L}^{2}\left(\eta_{1}+\eta_{2}\right)^{2}(1-\varepsilon) \varepsilon / h^{2} \omega^{2}}{2 \frac{e^{2}}{h \omega} E_{L}^{2}\left[\eta_{1} \varepsilon+\eta_{2}(1-\varepsilon)\right] \Delta f+2 \frac{e^{3}}{h^{2} \omega^{2}} \gamma E_{L}^{4}\left[\eta_{1} \varepsilon-\eta_{2}(1-\varepsilon)\right]^{2} \Delta f+4 k T \Delta f} .
\end{aligned}
$$

Compared with shot noise, thermal noise can often be ignored:

$$
S N R_{\text {balance }}=\frac{\left(\eta_{1}+\eta_{2}\right)^{2}(1-\varepsilon) \varepsilon E_{S}^{2} / h \omega}{\left(\eta_{1} \varepsilon+\eta_{2}(1-\varepsilon)\right) \Delta f+\left(\frac{e}{h \omega}\right) \gamma E_{1}^{2}\left[\eta_{1} \varepsilon-\eta_{2}(1-\varepsilon)\right]^{2} \Delta f} .
$$


Assume that the photosensitive area of one of the balanced detectors is $A_{1}$, and let $A_{1}$ approach to infinity or 0 , then the signal-to-noise ratio formula of detector 1 , namely ordinary heterodyne detection, is:

$$
\begin{aligned}
S N R_{1} & =\frac{\left(A_{1} \eta_{1}+\eta_{2}\right)^{2}(1-\varepsilon) \varepsilon E_{S}^{2} / h \omega}{\left(A_{1} \eta_{1} \varepsilon+\eta_{2}(1-\varepsilon)\right) \Delta f+\left(\frac{e}{h \omega}\right) \gamma E_{1}^{2}\left[A_{1} \eta_{1} \varepsilon-\eta_{2}(1-\varepsilon)\right]^{2} \Delta f} \\
& =\frac{\eta_{1}(1-\varepsilon) E_{S}^{2} / h \omega}{B W+\left(\frac{e}{h \omega}\right) \gamma E_{L}^{2} \eta_{1} \varepsilon \Delta f} .
\end{aligned}
$$

According to the SNR formula of Equation (20) for balanced coherent detection and the signal-to-noise ratio formula of Equation (21) for single source coherent detection, MATLAB software is used to carry out numerical simulation respectively. Some parameters in the SNR formula are as follows: the selected laser frequency is $150 \mathrm{THz}$, the photoelectric probe adopts InGaAs high-sensitivity PIN photoelectric diode, the band width $B W=1.0 \times 10^{6} \mathrm{~Hz}$, and the quantum efficiency is $\eta=1 \mathrm{~A} / \mathrm{W}$. It is assumed that the local oscillator power output by the fiber laser is $1 \mathrm{~mW}$, and the signal light power is $0.1 \%$ of the local oscillator power, i.e., $1 \mu \mathrm{W}$. The relative intensity noise coefficient $\gamma$ of the local oscillator light is $10^{3} \mathrm{~A}^{-1}$. Beam splitting ratio is variable, and the relation diagram of system noise and beam splitting ratio can be obtained.

It can be seen from Figure 3(a) that the maximum SNR of the balanced coherent detection system is $3.5 \mathrm{~dB}$ higher than that of the ordinary coherent detection. When the beam splitting ratio is 0.13 or 0.87 , the SNR of the balanced coherent detection is exactly equal to that of the ordinary coherent detection. When the beam splitting ratio is less than 0.13 or more than 0.87 , the SNR of the balanced coherent detection system is less than the SNR of the single source coherent detection.

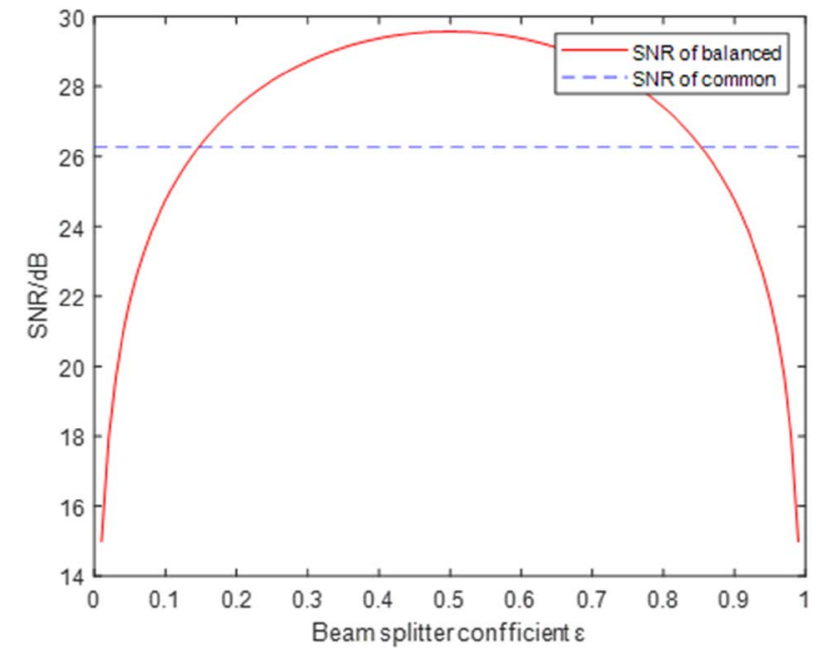

(a)

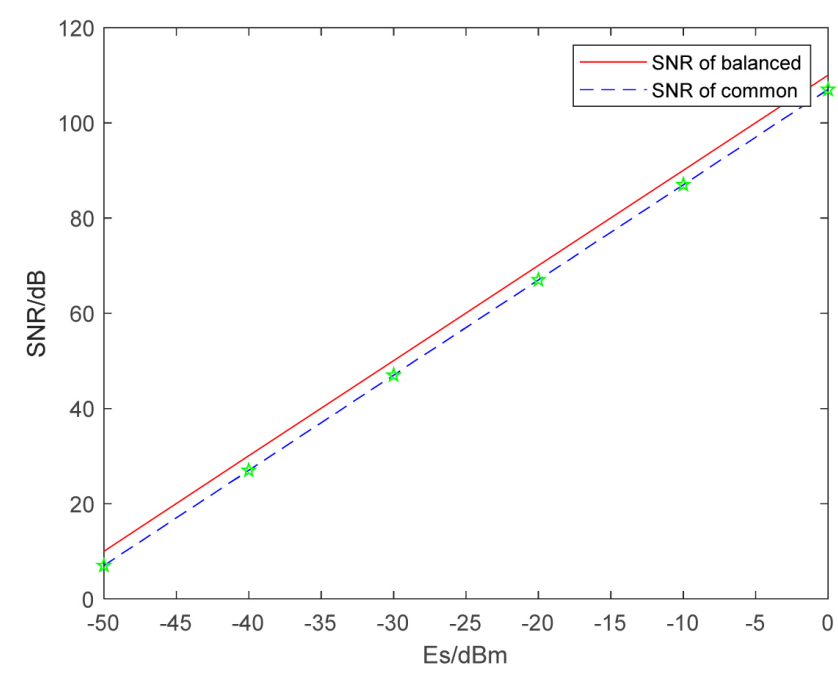

(b)

Figure 3. (a) Effect of beam splitting ratio on SNR; (b) Effect of signal optical power on SNR. 
If the above conditions remain unchanged and the signal optical power is reduced from $0 \mathrm{dBm}(1 \mathrm{~mW})$ to $-60 \mathrm{dBm}\left(10^{-6} \mathrm{~mW}\right)$, the SNR of the balanced coherent detection system and the SNR of the single source coherent detection are shown in the Figure 3(b).

It can be seen from the Figure 3(b) that the SNR of balanced detection is about $3 \mathrm{~dB}$ higher than that of ordinary heterodyne detection, under the condition that the signal optical power is taken as the variable.

\section{Simulation and Discussion}

\subsection{Balance Detection System Simulation}

The balance detection system model and the ordinary heterodyne detection system model are set as shown in Figure 4(a) and Figure 4(b). The local oscillator light power is set as $1 \mathrm{~mW}$, and the signal light carrier frequency is $100 \mathrm{MHz}$. The polarization direction of the signal light is strictly the same as the local oscillator light. The photoelectric probe adopts PIN photoelectric diode with band width $\mathrm{BW}=1.0 \times 10^{7} \mathrm{~Hz}$, and the value of quantum efficiency is $\eta=1 \mathrm{~A} / \mathrm{W}$. The signal light power is $0.1 \%$ of the local oscillator light power, that is, $1 \mathrm{uW}$, and the relative intensity noise coefficient $\gamma$ of the local oscillator light is $10^{3} \mathrm{~A}^{-1}$. THE beam splitting ratio is variable, and its simulation diagram is shown in Figure 5(a).

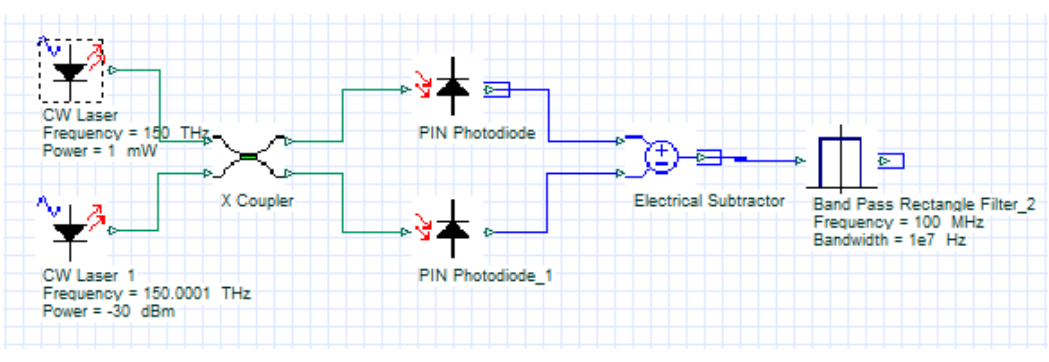

(a)

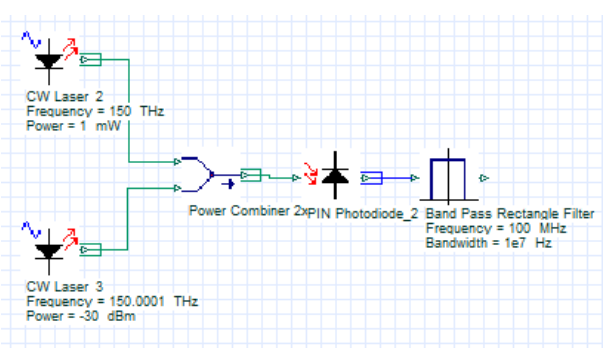

(b)

Figure 4. (a) Balance detection simulation diagram; (b) General heterodyne detection simulation diagram.

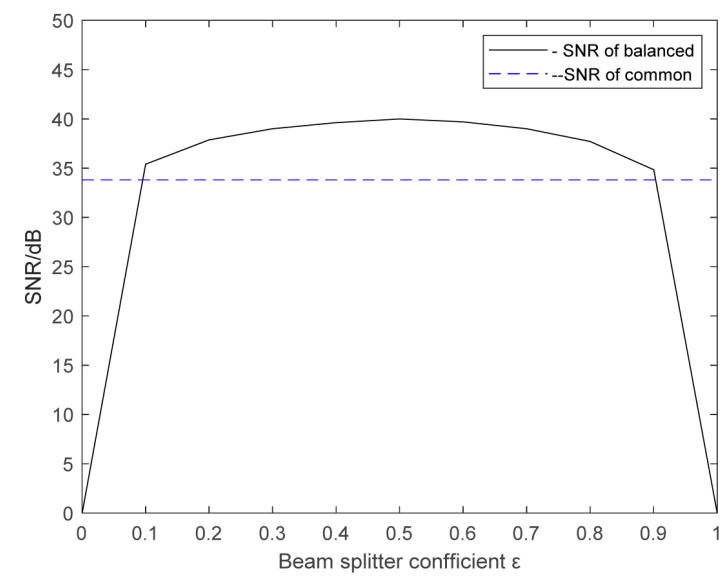

(a)

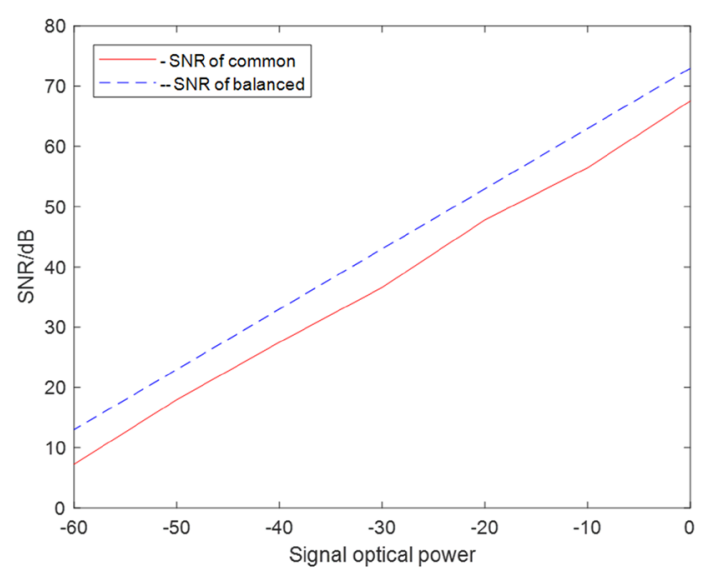

(b)

Figure 5. (a) The influence of beam splitting ratio on SNR; (b) Simulation diagram of the influence of signal optical power on SNR. 
It can be seen from Figure 5(a) that the maximum SNR of the balanced coherent detection system is $5 \mathrm{~dB}$ higher than that of the ordinary coherent detection. When the beam splitting ratio is 0.10 or 0.90 , the SNR of the balanced coherent detection is exactly equal to that of the ordinary coherent detection. When the beam splitting ratio is less than 0.10 or more than 0.90 , the SNR of the balanced coherent detection system is less than the SNR of the single source coherent detection. The simulation results are basically consistent with the SNR formula derived by us, which verifies the correctness of the derivation.

As shown in Figure 5(b), the SNR of balance detection is about $4 \mathrm{~dB}$ higher than that of ordinary heterodyne detection when the above conditions remain unchanged and the signal optical power is taken as variable, which is consistent with the theoretical results.

\subsection{Simulation Analysis of Double Balance Detection System}

The laser source is a continuous laser signal source, and the frequency is set to $150 \mathrm{THz}$. In order to make the difference frequency between the signal light and the local oscillator light exist, the frequency of the signal light is shifted by $1 \mathrm{G}$ relative to the local oscillator light, that is, the difference frequency of the two signals is $1 \mathrm{G}$. The local oscillator light power is $10 \mathrm{~mW}$, and the echo signal light power is $1 \mathrm{uW}$, both of which are linearly polarized light. Four PIN type photodetectors are selected, the response rate is $0.9 \mathrm{~A} / \mathrm{W}$, the dark current is $10 \mathrm{nA}$, and the noise characteristics of the detectors are the default of the system. The simulation diagram is shown in Figure 6.

Figure $7(a)$ and Figure $7(b)$ is the time domain simulation waveform diagram of the signals of system I and Q. It can be seen from the simulation results that the phase difference of the components of system $I$ and $Q$ is $\pi / 2$.

Rotate the $1 / 4$ wave plate and $1 / 2$ wave plate respectively, and the simulation results are shown in Figure 8 and Figure 9. When the 1/4 glass plate changes

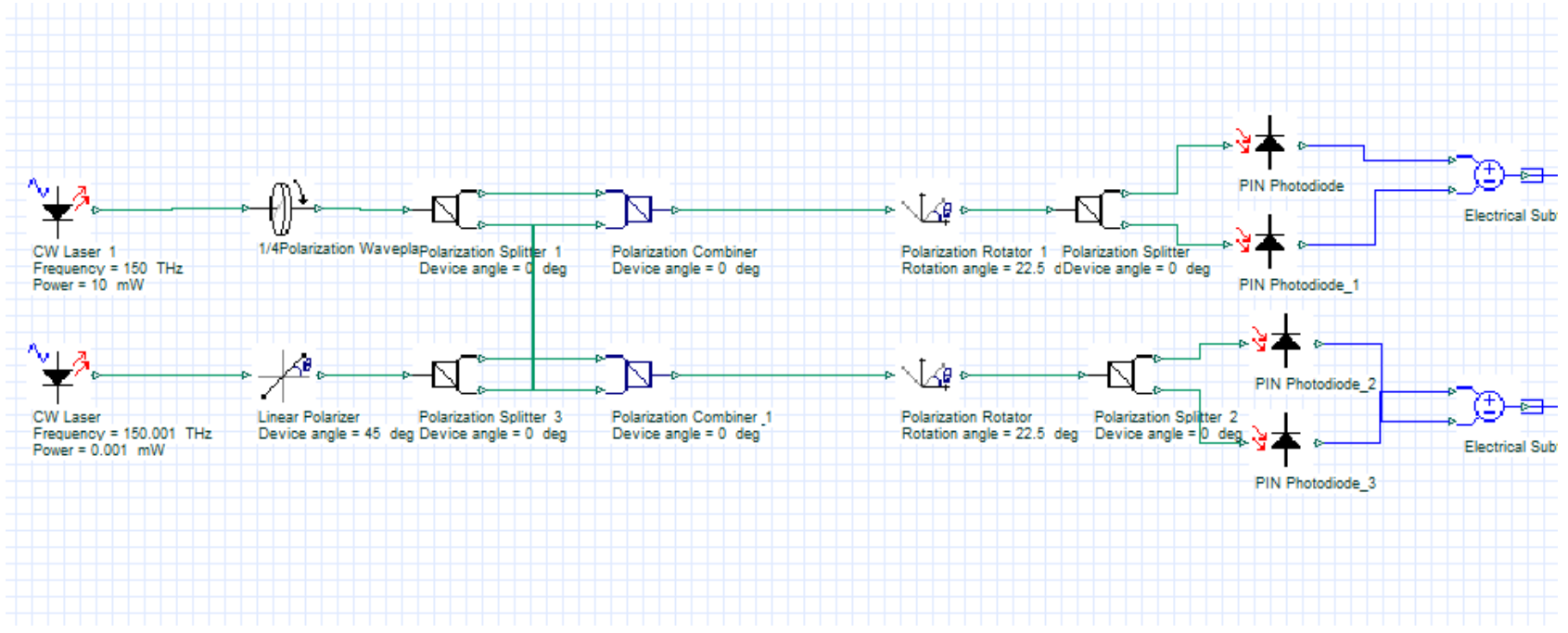

Figure 6. Simulation diagram of double balance detection. 


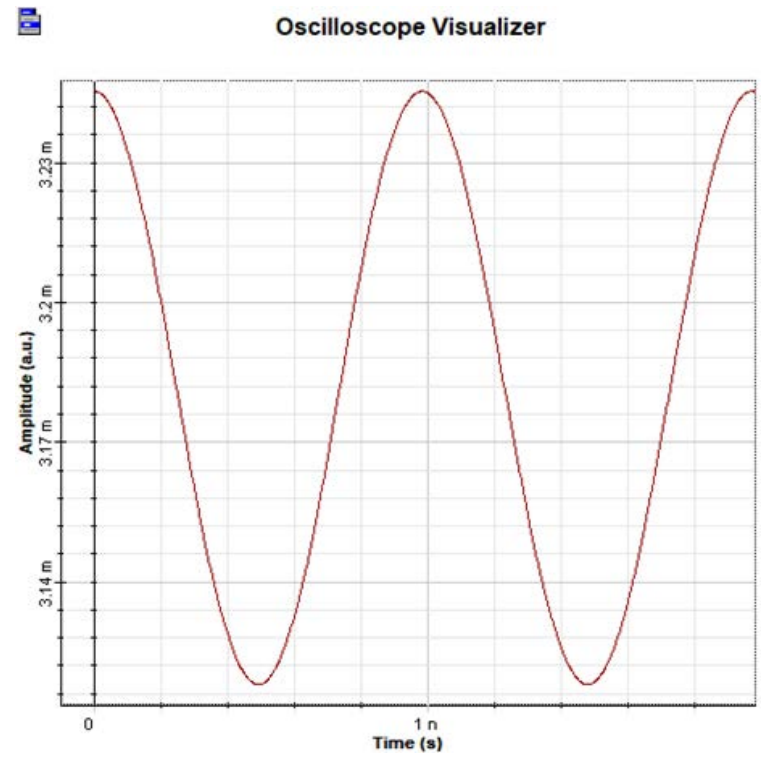

(a)

쿨

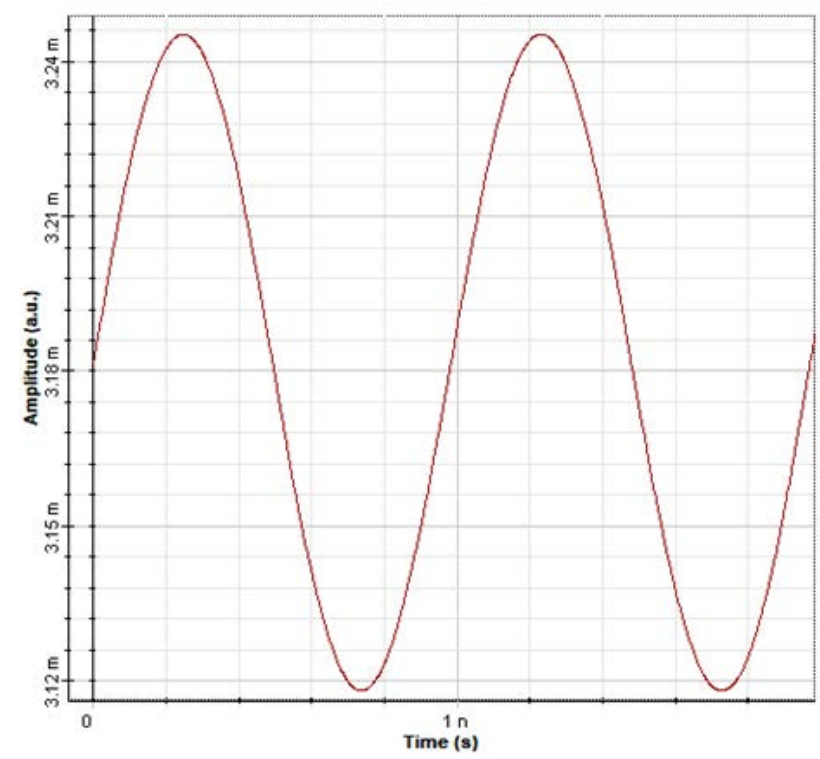

(b)

目

RF Spectrum Analyzer_1

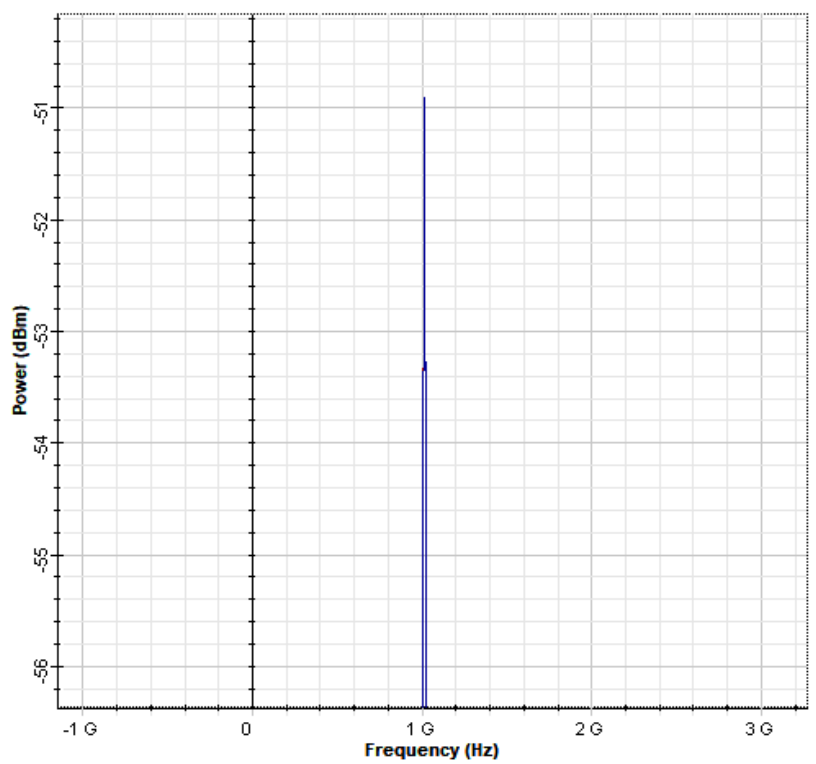

(c)

Figure 7. (a) is in-phase component (I) and (b) is quadrature component $(\mathrm{Q})$, the simulation results that the phase difference of the components of system $I$ and $Q$ is $\pi / 2$. (c) Difference frequency signal frequency domain waveform.

from a $45^{\circ}$ polarization angle to a $20^{\circ}$ polarization angle, the phase difference of signals in channel I and $\mathrm{Q}$ is not equal to $\pi / 2$.

After the $1 / 2$ glass slide was changed from $22.5^{\circ}$ to $30^{\circ}$, the SNR is reduced by $3 \mathrm{~dB}$.

Figure $7(c)$ is the frequency spectrum of the difference frequency signal. As can be seen from the figure, the difference frequency signal of $1 \mathrm{GHz}$ can be demodulated after differential processing, and the detected signal also has a better power value. 
룽

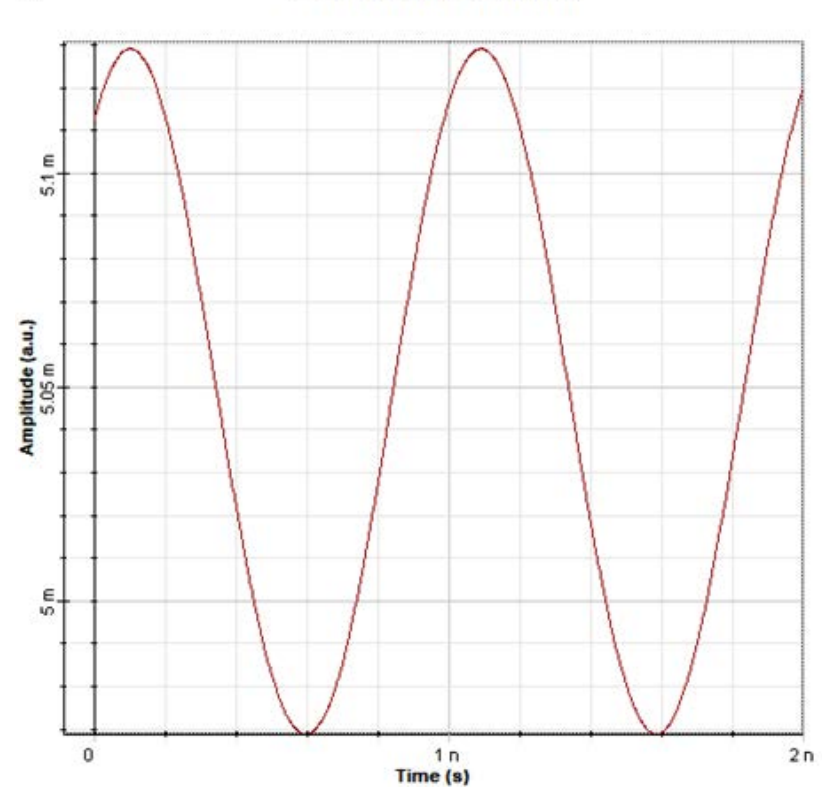

(a)

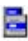

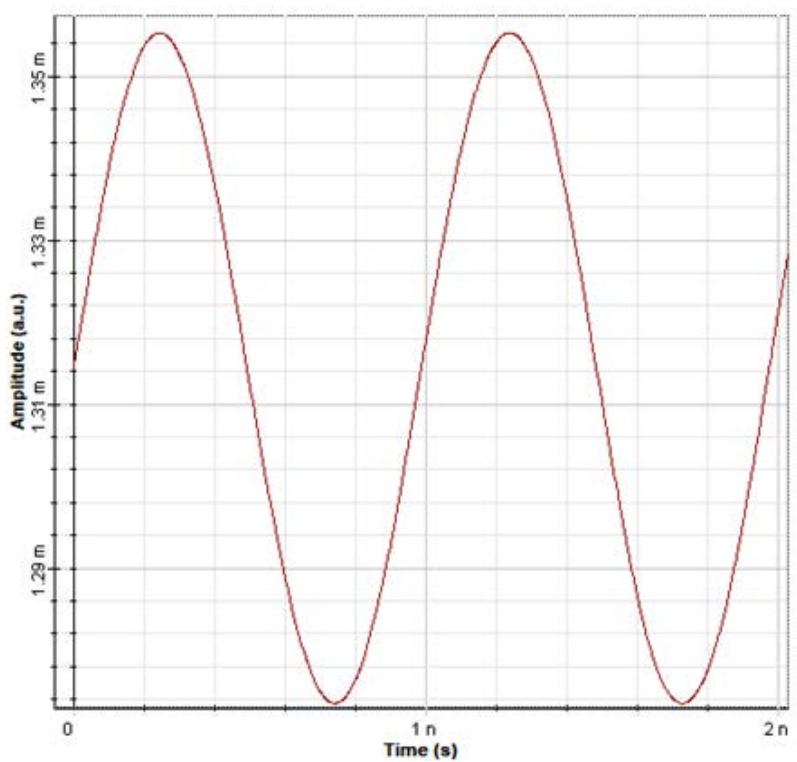

(b)

Figure 8. The $1 / 4$ glass plate changes from a $45^{\circ}$ polarization angle to a $20^{\circ}$ polarization angle, the phase difference of signals in channel (a) and (b) is not equal to $\pi / 2$ and the SNR was reduced by $2 \mathrm{~dB}$.

웅

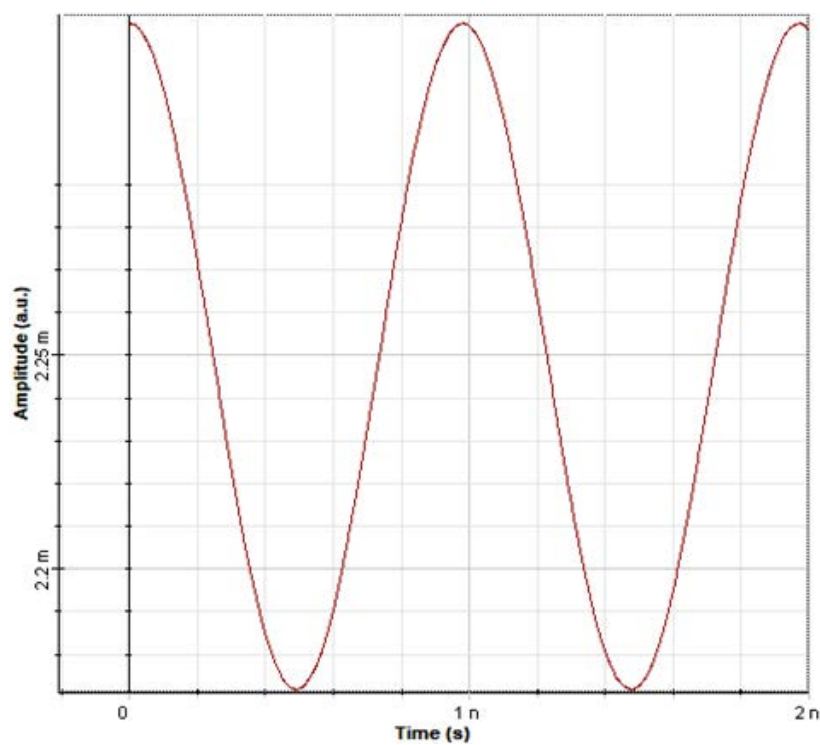

(a)
Oscilloscope Visualizer_1

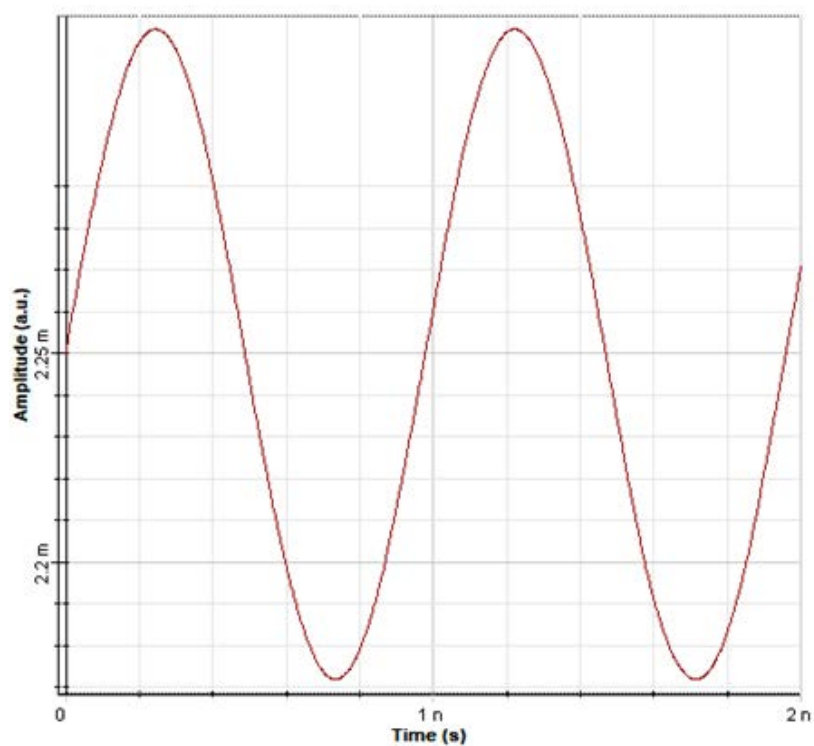

(b)

Figure 9. The $1 / 2$ glass slide was changed from $22.5^{\circ}$ to $30^{\circ}$, the SNR was reduced by $3 \mathrm{~dB}$ (from $41 \mathrm{~dB}$ To $38 \mathrm{~dB}$ ).

\section{Conclusion}

This paper analyzes the working principle of the balanced heterodyne detection system, establishes the simulation models of ordinary heterodyne detection, balance detection and double balance detection system are built by OptiSystem. The simulation conclusion is consistent with the mathematical deduction. In the 
double balanced detection simulation, from the different simulation results obtained from the rotation after the wave plate, we can draw the following conclusion. When $1 / 4$ wave plate is $45^{\circ}$ and the two $1 / 2$ wave plates are at an angle of $22.5^{\circ}$ with the incident light (corresponding to the splitting ratio similar to 0.5 ), the detected signal can obtain the Maximum power, the conclusion also studies double balanced heterodyne detection system of the best SNR conditions.

\section{Funding}

This work was supported by the National Natural Science Foundation of China under Grant 61875008.

\section{Conflicts of Interest}

The authors declare no conflicts of interest regarding the publication of this paper.

\section{References}

[1] Svitek, R. and Raman, S. (2005) DC Offsets in Direct-Conversion Receivers: Characterization and Implications. Microwave Magazine IEEE, 6, 76-86. https://doi.org/10.1109/MMW.2005.1511916

[2] Eang, S.H., Choi, H.D., Yoon, S. and Cho, K. (2011) Application of Heterodyne Double Pass Interferometer on the Readout Sensor for a Biochemical Fluidic Channel. IEEE. https://doi.org/10.1109/IQEC-CLEO.2011.6194124

[3] Onori, D., Laghezza, F., Scotti, F., Bartocci, M. and Ghelfi, P. (2016) A Direct-Conversion RF Scanning Receiver Based on Photonics. 2016 IEEE/MTT-S International Microwave Symposium, San Francisco, 22-27 May 2016. https://doi.org/10.1109/MWSYM.2016.7540327

[4] Zhu, X., Jin, T., Chi, H., Tong, G. and Lai, T. (2018) A Coherent Photonic RF Scanning Receiver Based on a Flat Optical Frequency Comb. Optics Communications, 421, 41-45. https://doi.org/10.1016/j.optcom.2018.03.054

[5] Jing, L., Xiao, W., Zhu, X. and Zhang, C., et al. (2010) A High Sensitivity and Wide Dynamic Range Zero-If RF Receiver for Cognitive Radio Application. Journal of Electronics, 27, 696-700. https://doi.org/10.1007/s11767-011-0499-7

[6] Chan, V.W.S. (2006) Free-Space Optical Communications. Journal of Lightwave Technology, 24, 4750-4762. https://doi.org/10.1109/JLT.2006.885252

[7] Seimetz, M. and Weinert, C.M. (2006) Options, Feasibility, and Availability of $2 \times 4$ $90^{\circ}$ Hybrids for Coherent Optical Systems. Journal of Lightwave Technology, 24, 1317-1322. https://doi.org/10.1109/JLT.2005.863251

[8] Savory, S.J. (2010) Digital Coherent Optical Receivers: Algorithms and Subsystems. IEEE Journal of Selected Topics in Quantum Electronics, 16, 1164-1179. https://doi.org/10.1109/JSTQE.2010.2044751

[9] Seimetz, M. (2009) High-Order Modulation for Optical Fiber Transmission. Springer Berlin Heidelberg. https://doi.org/10.1007/978-3-540-93771-5

[10] Lygagnon, D.S., Katoh, K. and Kikuchi, K. (2005) Unrepeated 210-km Transmission with Coherent Detection and Digital Signal Processing of 20-Gb/s QPSK Signal. https://doi.org/10.1007/978-3-540-93771-5

[11] Yu, J., Singh, U.N., Barnes, J.C., Barnes, N.P. and Petros, M. (2001) High-Energy 
2- $\mu \mathrm{m}$ Laser for Multiple Lidar Applications. Proceedings of SPIE-The International Society for Optical Engineering, 4153, 70-77. https://doi.org/10.1117/12.417025

[12] Frehlich, R., Hannon, S.M. and Henderson, S.W. (1994) Performance of a 2-m Coherent Doppler Lidar for Wind Measurements. J. Atomos. Oceanic Technol, 11, 1517-1528. https://doi.org/10.1175/1520-0426(1994)011<1517:POACDL $>2.0 . C O ; 2$

[13] Wang, C., Amzajerdian, F. and Gao, C.Q. (2009) Investigation of Balanced Detection and Receiver for Coherent Lidar. Proceedings of SPIE-The International Society for Optical Engineering, 7382, 73820I. https://doi.org/10.1117/12.829459

[14] Chen, B., Fan, Y. and Gao, Y. (2020) Wideband Coherent Optical RF Channelizer with Image-Reject Down-Conversion. Asia Communications and Photonics Conference. https://doi.org/10.1364/ACPC.2020.M4A.335

[15] Park, Y. and Cho, K. (2011) Heterodyne Interferometer Scheme Using a Double Pass in an Acousto-Optic Modulator. Optics Letters, 36, 331-333.

https://doi.org/10.1364/OL.36.000331 